\title{
Comorbid chronic diseases and their associations with quality of life among gynecological cancer survivors
}

\author{
Ji-Wei Wang ${ }^{1 \dagger}$, Li Sun ${ }^{1 \dagger}$, Jiang Li ${ }^{2}$, Xiao-Huan Cong ${ }^{1}$, Xue-Fen Chen ${ }^{1}$, Zheng Tang ${ }^{1}$, Dong-Hui Yu ${ }^{3}$, Tian-Rui Zhang ${ }^{1}$,
} Zheng-Nian Luo ${ }^{4}$, Zheng-Ping Yuan ${ }^{5}$ and Jin-Ming Yu ${ }^{1 *}$

\begin{abstract}
Background: Many gynecological cancer survivors (GCS) have comorbid chronic diseases (CCD). This study was to estimate the impacts of CCD on quality of life (QOL) in GCS.

Methods: We collected cross-sectional self-reported survey data from 598 GCS between April and July 2013, in Shanghai, China. All the subjects were asked to complete a questionnaire containing the European Organization for Research and Treatment quality of life version 3 questionnaire (EORTC QLQ-C30) and questions on socio-demographic characteristics and CCD. In order to mitigate the bias caused by confounding factors, multiple linear models were employed to calculate adjusted means of QOL scores.

Results: Approximately three-quarters of subjects reported at least one CCD. The highest overall prevalence of all CCD was found in endometrial cancer survivors. Subjects with CCD generally reported lower scores for most EORTC QLQ-C30 scales when compared to subjects without CCD, indicating poorer QOL, particularly for cardiovascular diseases, respiratory diseases, digestive diseases, and musculoskeletal disease.
\end{abstract}

Conclusions: The CCD are common health problems among GCS. CCD have significantly negative influence on QOL, and GCS with CCD generally reported lower QOL scores. These findings suggested comprehensive cares for GCS.

Keywords: Gynecological cancer survivors, Quality of life, Comorbid chronic diseases, Cancer care

\section{Background}

Gynecologic cancers are the third most common female cancer, occurring in about 1 in 20 women worldwide [1], and are a major source of mortality and morbidity [2]. Due to population expansion and increasing proportion of the elderly population, the number of gynecological cancers in China is rapidly increasing [3]. With the exception of ovarian cancer, most gynecological cancer diagnoses are associated with good survival rates [4-6]. Thus, more and more Chinese women who will be longterm gynecologic cancer survivors (GCS) are added to the population each year [3].

\footnotetext{
* Correspondence: jmy@fudan.edu.cn

${ }^{\dagger}$ Equal contributors

'School of public health and Key Laboratory of Public Health Safety, Fudan University, 30 Dong-An Road, Shanghai 200032, China

Full list of author information is available at the end of the article
}

Despite longer survival time, GCS may continuously live with conditions such as long-term and late physical, psychosocial and sexual effects of gynecological cancer and treatment $[7,8]$, as well as associated comorbid chronic diseases (CCD) that influence their survival and quality of life (QOL). Assessment of QOL to monitor and support long-term GCS for both medical and psychologic consequences of their cancer treatment and rehabilitation is imperative. A few studies to date have examined the impact of CCD on gynecologic cancer survival, and generally indicated that there was poorer survival among GCS with CCD, compared with those without CCD [9-13].

Although much is known about survival rates and complications of therapy for gynecologic cancers, the issue of QOL has been addressed only recently. Gynecologic cancers pose special risks for QOL $[14,15]$. Given the challenges and changes that women must face after 
a diagnosis of gynecologic cancer, QOL is an especially pertinent issue that needs attention. Evaluating and addressing QOL issues have been an important part of the whole package of modern medical care in GCS [16].

The potential QOL deficits associated with GCS who has CCD may be quite large. However, despite the recognition of the link between CCD and GCS, very little has been known about the impact of CCD on QOL in these patients. The objectives of this study were to examine the prevalence of CCD and to estimate the impact of CCD on QOL in the following groups: 1) GCS with CCD compared to GCS without CCD, and 2) GCS with one kind of CCD compared to GCS without it. A better understanding of the effects of CCD on QOL is needed to improve quality of care among GCS.

\section{Methods}

\section{Recruitment}

From April to July 2013, we consecutively contacted GCS in multi-community cancer rehabilitation centers, all of which were affiliated Shanghai Cancer Rehabilitation Club, Shanghai, China- a non-government organization exclusively for cancer survivors. It covered all the 17 districts in Shanghai and has about 13500 cancer survivor members in Shanghai by the end of 2012. This peer support group aims at improving survivors' QOL. The SCRC regularly offers rehabilitation activities including physical exercise, relaxation training, counseling, psychotherapy and a variety of leisure activities [17].

There were 899 GCS registered in the SCRC by the end of 2012. All members who intended to continue to participate in the SCRC were required to register annually, including the former members and new members. The survey invitations were sent through short text messages and/or phone calls to all 899 GCS. Among all these GCS, 70 were unable to reach possibly because of deaths, migration or refusing to response; 231 declined to participate the survey either because they had no time, or because their health status or literacy ability was too poor. Finally, 598 participated in this survey and formed our final sample, and completed the selfadministered questionnaire, which normally took 40-60 minutes. Ethical approval to conduct this study was granted by the Medical Research Ethics Committee of the School of Public Health, Fudan University (Protocol number RB \#2013-04-0450). Written informed consent was obtained from each participant.

\section{Instruments}

\section{Socio-demographic characteristics}

We collected information on age, gender, educational level, Body Mass Index, and other socio-demographic information.

\section{Comorbid chronic diseases}

Participants were asked to indicate either "yes" or "no" on a list of CCD including hypertension, diabetes mellitus, heart and cardiovascular diseases, respiratory diseases, digestive diseases, musculoskeletal diseases.

\section{EORTC QLQ-C30}

The EORTC QLQ-C30 (version 3.0) was designed and validated to assess QOL [18]. It is a 30-item patient selfrating questionnaire that can be applied to all cancer patients. The EORTC QLQ-C30 is composed of a global health status/QOL-score, five (multi-item) function scales (physical, role, social, emotional and cognitive functions), three multi-item symptom scales (fatigue, pain, nausea), and five single items (dyspnea, insomnia, appetite loss, constipation, diarrhea). A final item evaluates the perceived economic consequences of the disease. Each item has four response options: (1) "not at all", (2) "a little", (3) "quite a bit", and (4) "very much" except the two items of the global health-status/quality of life scale which have response options ranging from (1) "very poor" to (7) "excellent". According to the guidelines provided by the EORTC, all scores of the QLQ-C30 were transformed linearly so that all scales ranged from 0 to 100. A higher score for functional scales indicates a healthier level of functioning, and a higher score for global health status/ QOL indicates a higher QOL. A higher score on the symptom scale and on single items indicates a higher level of symptoms or problems [19].

\section{Statistical analysis}

Standard statistical methods were used to examine potential differences in covariates including chi-squared tests for categorical variables and one-way analysis of variance (ANOVA) for continuous variables followed by Bonferroni post hoc tests. CCD was defined in the statistical analyses as a dichotomous variable (yes/no). Multivariate linear regression models were used to compute regression coefficients $(\beta)$ and associated $95 \%$ confidence intervals as estimates of the mean difference of QOL scores associated with the presence of absence of $\mathrm{CCD}$, adjusting for potential confounding variables. The following potentially confounding variables were included in all regression models: age (continuous), Body Mass Index(continuous), years since diagnosis(continuous), household income (continuous), education (less than junior high school, junior high school or Junior high school), current marital status (married/living with partner or divorced/widowed/ separated/single), and treatment(surgery, chemotherapy, and/or traditional Chinese medicine). Tests for trend were performed by entering the categorical variables as continuous parameters in the model. Statistical tests were based on a two- 
Table 1 Socio-demographic and treatment characteristic of GCS ( $N=598)$

\begin{tabular}{|c|c|c|c|c|c|c|c|c|c|}
\hline \multirow[t]{2}{*}{ Characteristics } & \multicolumn{2}{|l|}{ Total } & \multicolumn{2}{|c|}{ Cervical cancer } & \multicolumn{2}{|c|}{ Ovarian cancer } & \multicolumn{2}{|c|}{ Endometrial cancer } & \multirow[t]{2}{*}{$P$} \\
\hline & $N=598$ & $\%$ & $N=224$ & $\%$ & $N=299$ & $\%$ & $N=75$ & $\%$ & \\
\hline Age(year) & & & & & & & & & 0.000 \\
\hline$<50$ & 88 & 14.7 & 56 & 25.0 & 23 & 7.7 & 9 & 12.0 & \\
\hline $50-59$ & 251 & 42.0 & 94 & 42.0 & 131 & 43.8 & 26 & 34.7 & \\
\hline $60-69$ & 195 & 32.6 & 47 & 21.0 & 118 & 39.5 & 30 & 40.0 & \\
\hline$\geq 70$ & 64 & 10.7 & 27 & 12.1 & 27 & 9.0 & 10 & 13.3 & \\
\hline Body Mass Index & & & & & & & & & 0.044 \\
\hline$<18.5$ & 29 & 4.8 & 14 & 6.3 & 11 & 3.7 & 4 & 5.3 & \\
\hline $18.5-24$ & 360 & 60.2 & 137 & 61.2 & 190 & 63.5 & 33 & 44.0 & \\
\hline $25-29$ & 185 & 30.9 & 66 & 29.5 & 87 & 29.1 & 32 & 42.7 & \\
\hline$\geq 30$ & 24 & 4.0 & 7 & 3.1 & 11 & 3.7 & 6 & 8.0 & \\
\hline Years since diagnosis & & & & & & & & & 0.001 \\
\hline$<2$ & 68 & 11.4 & 34 & 15.2 & 29 & 9.7 & 5 & 6.7 & \\
\hline $2-5$ & 175 & 29.3 & 82 & 36.6 & 72 & 24.1 & 21 & 28.0 & \\
\hline$\geq 6$ & 355 & 59.4 & 108 & 48.2 & 198 & 66.2 & 49 & 65.3 & \\
\hline Marital status & & & & & & & & & 0.433 \\
\hline Married/with partner & 505 & 84.4 & 189 & 84.4 & 249 & 83.3 & 67 & 89.3 & \\
\hline Divorced/widowed/separated/ single & 93 & 15.6 & 35 & 15.6 & 50 & 16.7 & 8 & 10.7 & \\
\hline Education & & & & & & & & & 0.015 \\
\hline Less than junior high school & 61 & 10.2 & 33 & 14.7 & 26 & 8.7 & 2 & 2.7 & \\
\hline Junior high school & 243 & 40.6 & 94 & 42.0 & 117 & 39.1 & 32 & 42.7 & \\
\hline More that junior high school & 294 & 49.2 & 97 & 43.3 & 156 & 52.2 & 41 & 54.7 & \\
\hline Household income(yen/month) & & & & & & & & & 0.000 \\
\hline$<2000$ & 187 & 31.3 & 98 & 43.8 & 74 & 24.7 & 15 & 20.0 & \\
\hline $2000-4000$ & 322 & 53.8 & 94 & 42.0 & 180 & 60.2 & 48 & 64.0 & \\
\hline$>4000$ & 89 & 14.9 & 32 & 14.3 & 45 & 15.1 & 12 & 16.0 & \\
\hline \multicolumn{10}{|l|}{ Treatment } \\
\hline Surgery & 532 & 89.0 & 197 & 87.9 & 264 & 88.3 & 71 & 94.7 & 0.240 \\
\hline Radiotherapy & 179 & 29.9 & 99 & 44.2 & 55 & 18.4 & 25 & 33.3 & 0.000 \\
\hline Chemotherapy & 435 & 72.7 & 137 & 61.2 & 254 & 84.9 & 44 & 58.7 & 0.000 \\
\hline Traditional Chinese Medicine & 293 & 49.0 & 80 & 35.7 & 145 & 48.5 & 39 & 52.0 & 0.005 \\
\hline No. of comorbid chronic diseases & & & & & & & & & 0.004 \\
\hline 0 & 138 & 23.1 & 65 & 29.0 & 61 & 20.4 & 12 & 16.0 & \\
\hline 1 & 138 & 23.1 & 59 & 26.3 & 68 & 22.7 & 11 & 14.7 & \\
\hline 2 & 119 & 19.9 & 40 & 17.9 & 64 & 21.4 & 15 & 20.0 & \\
\hline$\geq 3$ & 203 & 33.9 & 60 & 26.8 & 106 & 35.5 & 37 & 49.3 & \\
\hline \multicolumn{10}{|l|}{ Comorbid chronic disease } \\
\hline Hypertension & 184 & 30.8 & 60 & 26.8 & 95 & 31.8 & 29 & 38.7 & 0.135 \\
\hline Diabetes & 106 & 17.7 & 25 & 11.2 & 59 & 19.7 & 22 & 29.3 & 0.001 \\
\hline Heart and Cardiovascular & 147 & 24.6 & 49 & 21.9 & 75 & 25.1 & 23 & 30.7 & 0.298 \\
\hline Respiratory diseases & 49 & 8.2 & 17 & 7.6 & 22 & 7.4 & 10 & 13.3 & 0.221 \\
\hline Digestive diseases & 297 & 49.7 & 104 & 46.4 & 149 & 49.8 & 44 & 58.7 & 0.185 \\
\hline musculoskeletal diseases & 191 & 31.9 & 68 & 30.4 & 97 & 32.4 & 26 & 34.7 & 0.760 \\
\hline
\end{tabular}


tailed probability with a significance level of 0.05 . All statistical analyses were performed using SAS.9.3 (SAS Institute, Cary, NC).

\section{Results}

The characteristics of the study sample

All 899 GCS registered in the SCRC were invited to participate, of whom 598 (66.5\%) responded to the survey. The characteristics of the participants are shown in Table 1. Forty-three percent of the sample was 60 years old or above. Majority of them were married (84.4 \%) and had at least one CCD (76.9 \%). Nearly $35 \%$ of the participants were overweight or obese. Some statistically significant differences between cancer types were noted.

In terms of the types of CCD, the proportion of GCS who suffered from digestive diseases $(49.7 \%)$ was the highest. Other CCD are listed according to their prevalence as follows: hypertension (30.8\%), diabetes mellitus $(17.7 \%)$, heart and cardiovascular diseases $(24.6 \%)$, respiratory diseases $(8.2 \%)$, digestive diseases (49.7\%), musculoskeletal diseases (31.9 \%). Meanwhile, the highest overall prevalence of all CCD was found in endometrial cancer survivors. The prevalence of diabetes was significantly different between cancer types.

\section{The influence of comorbid chronic diseases on EORTC QLQ-C30 scores}

The influences of CCD on EORTC QLQ-C30 scores are presented in Table 2. After adjusting for the influence of the socio-demographic variables, subjects with selfreported CCD generally reported lower scores for most EORTC QLQ-C30 scales when compared to subjects without these CCD, indicating poorer QOL. The influences of heart and cardiovascular diseases, respiratory diseases and musculoskeletal illnesses on EORTC QLQC30 scores were of a similar magnitude and were larger than the influence of hypertension, diabetes or digestive diseases.

\section{Discussion}

Based on our data, the prevalence of the CCD including hypertension, diabetes mellitus, heart and cardiovascular diseases, respiratory diseases, digestive diseases, musculoskeletal diseases was quite high among GCS. Seventy seven percent of GCS had at least one of the CCD considered in this study. Furthermore, the prevalence of multiple CCD was significant in endometrial cancer survivors, with nearly half of them suffering from two or more CCD. This study indicated that QOL scores of

Table 2 Quality of life for subjects with and without comorbid chronic diseases after adjusting for the influence of the sociodemographic variables

\begin{tabular}{|c|c|c|c|c|c|c|c|}
\hline & $\begin{array}{l}C C D, n=460 \\
(\text { no } C C D, n=138 \text { ) }\end{array}$ & $\begin{array}{l}\text { HPT, } n=184 \\
\text { (no HPT, } n=414 \text { ) }\end{array}$ & $\begin{array}{l}\text { DM, } n=106 \\
\text { (no DM, } n=492 \text { ) }\end{array}$ & $\begin{array}{l}\mathrm{HD}, n=147 \\
(\text { no } H D, n=451)\end{array}$ & $\begin{array}{l}\mathrm{RD}, n=49 \\
\text { (no RD, } n=549 \text { ) }\end{array}$ & $\begin{array}{l}D D, n=297 \\
(\text { no } D D, n=301)\end{array}$ & $\begin{array}{l}S D, n=191 \\
\text { (no SD, } n=407 \text { ) }\end{array}$ \\
\hline \multicolumn{8}{|c|}{ EORTC QLQC-30 } \\
\hline PF & $-2.50(83.46)$ & $-5.43(83.09) * *$ & $-3.87(82.20)$ & $-6.95(82.95) * *$ & $-7.79(82.07) *$ & $-1.46(82.28)$ & $-5.58(83.31) * *$ \\
\hline RF & $-3.66(92.49)$ & $-4.73(90.99) *$ & $-6.44(90.77) *$ & $-8.21(91.32) * *$ & $-14.94(90.71) * * *$ & $-1.50(90.41)$ & $-4.72(91.13) *$ \\
\hline CF & $-5.81(87.86) *$ & $-3.24(84.22) * *$ & $-4.28(84.05)$ & $-5.94(84.51) *$ & $-9.76(83.99) * *$ & $-4.88(85.93) * *$ & $-8.99(86.20) * * *$ \\
\hline EF & $-5.71(81.92) *$ & $-3.93(78.56) * *$ & $-4.88(78.30) *$ & $-5.70(78.60) *$ & $-7.00(77.93) *$ & $-5.02(80.15)^{* *}$ & $-6.60(79.56) * *$ \\
\hline SF & $-5.71(80.59)$ & $-5.69(77.75) *$ & $-1.90(76.42)$ & $-7.61(77.67) *$ & $-15.34(77.22) * *$ & $-4.81(78.70) *$ & $-8.67(78.90) * *$ \\
\hline QL & $-7.22(65.93) *$ & $-4.37(61.50) * *$ & $-5.69(61.26)$ & $-9.25(62.16) * *$ & $-13.19(61.20)^{* *}$ & $-4.00(62.40)$ & $-11.26(63.89) * * *$ \\
\hline FA & $8.82(23.22) * *$ & $5.00(28.72) *$ & $2.84(29.68)$ & $10.09(28.07) * * *$ & $9.42(29.49) *$ & $8.55(25.52) * * *$ & $7.96(27.60) * *$ \\
\hline NV & $0.39(3.20)$ & $1.60(3.04)$ & $2.79(3.00) *$ & $3.29(2.82) * *$ & $3.48(3.25)$ & $-0.58(3.83)$ & $1.29(3.09)$ \\
\hline PA & $3.22(15.29)$ & $6.94(15.79) * *$ & $2.83(17.32)$ & $9.40(15.86) * * *$ & $13.43(16.83) * * *$ & $1.04(17.27)$ & $12.85(13.64) * * *$ \\
\hline DY & $6.90(9.47) * *$ & $1.79(14.40)$ & $1.93(14.58)$ & $10.63(12.69) * * *$ & $21.24(13.34) * * *$ & $4.28(12.59) *$ & $4.38(13.50) *$ \\
\hline SL & $7.99(14.90) *$ & $2.43(20.51)$ & $3.46(20.59)$ & $10.13(19.09) * *$ & $16.22(20.01) * * *$ & $5.92(17.99) *$ & $9.11(18.25)$ \\
\hline AP & $3.89(6.36)$ & $2.83(8.61)$ & $2.69(8.95)$ & $3.76(8.65)$ & $9.93(8.70) * *$ & $-0.03(9.46)$ & $6.95(7.17) * *$ \\
\hline $\mathrm{CO}$ & $6.05(7.88) *$ & $0.29(12.59)$ & $4.86(11.78)$ & $7.71(11.05) * *$ & $12.11(11.76) * *$ & $3.29(10.87)$ & $3.70(11.46) * *$ \\
\hline Dl & $4.39(5.08)$ & $0.06(8.54)$ & $0.48(8.47)$ & 1.87(8.16) & $11.55(7.69) * * *$ & $1.53(7.72)$ & $2.95(7.59)$ \\
\hline $\mathrm{FI}$ & 4.82(30.89) & $5.34(33.13)$ & $5.50(33.69)$ & $9.67(32.67) *$ & $13.54(33.69)$ * & $4.72(32.12)$ & $2.71(33.82)$ \\
\hline
\end{tabular}

1.Quality of life questionnaire abbreviation: Physical Functioning PF;Role Functioning, RF;Cognitive Functioning, CF;Emotional Functioning, EF;Social Functioning, SF;Global Health,QL; Fatigue, FA; Nausea and vomiting, NV; Pain, PA; Dyspnoea, DY; Insomnia, SL; Appetite loss, AP; Constipation, CO; Diarrhoea, DI; financial difficultis, FI. Social Functioning, SF

2. Comorbid chronic diseases abbreviation: Comorbid chronic diseases, CCD; hypertension, HPT; diabetes mellitus, DM; heart and cardiovascular diseases, HCD; respiratory diseases, RD; digestive diseases, DD; musculoskeletal diseases, MD

3. The difference in mean score of quality of life between GCS with and without CCD (mean score of quality of life among GCS without CCD)

4. Multiple linear regression, adjusted for influence of gender, age, BMI, education, household income, time after diagnosis, treatment.

5. * $P<0.05, * * P<0.01,{ }^{* * *} P<0.001$ 
GCS with CCD were lower than those without reporting the corresponding CCD.

Previous studies have shown that patient demographic characteristics such as age, sex, marital status, and education are important factors associated with cancer QOL $[20,21]$, and the main purpose of this study was to understand the impact of CCD on QOL; therefore, multiple linear regression models were used to control for the effects of socio-demographic characteristic.

Cancer survivors with CCD have clinical and health care needs that may differentiate them from persons without $\mathrm{CCD}$. Evidence indicates that persons with one CCD are more likely to have other CCD $[22,23]$. The GCS in our study reported that more than half of them have more than one CCD. Moreover, cancer survivors with severe CCD may have more rapid declines in health status and a greater likelihood of disability and mortality [24-26]. In China, the management of chronic diseases and cancer was often fragmented, for the management of cancer was more focused on cancer recurrence and metastasis. The significant impact of CCD on the health and QOL of cancer patients, however, was easily overlooked.

Our study indicated that the CCD impacts the QOL of GCS. Added to known cancer-specific vulnerabilities, the increased risk of CCD makes the need for comprehensive care programs for GCS even more urgent. Given the challenges and changes that women must face after a diagnosis of gynecologic cancer, QOL is an especially pertinent issue that we ought to focus on. Understanding the long-term physical and psychosocial effects of cancer and CCD can help gynecologic patients and their families better deal with potential health risks associated with being a cancer survivor. CCD prevention and control will help improve the QOL and the overall health of GCS.

Obesity is a serious health problem which has significant impact on the incidence and treatment of the gynecologic cancers, especially for endometrial cancer [27, 28]. Obese women with cancers have decreased survival rate which may be due to disease-specific reasons [29], the result of comorbid illnesses [30], or response to treatment [31]. In our study, nearly $35 \%$ of subjects were overweight and obese. In a nationally representative sample of 15,540 Chinese middle-aged and elderly adults, the prevalence of overweight and obesity were $36.9 \%$ in men and $31.1 \%$ in women, respectively [32]. Obviously, the prevalence of overweight and obese in GCS was very high. The China National Diabetes and Metabolic Disorders Study, conducted from June 2007 to May 2008, revealed that $8.8 \%$ of all women aged 20 years or older had diabetes, $0.51 \%$ had coronary heart disease, $0.60 \%$ had had a stroke and $1.10 \%$ had cardiovascular disease [33]. Comparing with the general Chinese population, the prevalence of CCD in our cohort was also very high. Meanwhile, the highest overall prevalence of all CCD was found in endometrial cancer survivors.

High calorie intake and little physical activity are associated with obese and often result in many other comorbidities e.g., diabetes mellitus, hypertension, dyslipidemia, coronary heart disease, gallbladder disease, arthritis and constipation [34]. The association between the CCD and QOL among GCS warrants changes in the lifestyle for patients and also challenges old paradigms that oncology's work is done after treatment. Collaborative chronic disease management models may be particularly appropriate in this regard, which emphasizes continuity of long-term care and a relationship between patient and provider in which the patient is empowered and takes an active role in their ongoing care such as making lifestyle changes $[35,36]$.

Our study has several limitations that merit discussion. Firstly, all the subjects were recruited from the Cancer Rehabilitation Club. The most common reason that GCS attended the SCRC perhaps was that they were more unwell and feel a need for support and rehabilitation. Possibly, the QOL of our sample was worse compared with the overall population of Chinese GCS. It was also necessary that comparing the difference of QOL between the more broad population of Chinese GCS and other types of cancer survivors, moreover, and women of the general population who have CCD for future studies. Second, information on cancer staging was not collected in this study. Examining the association between cancer staging and QOL will be the focus of our future study. Finally, the validity of self-reported CCD could be questionable [37], however, in our study, we made it clear to the respondents that the CCD must be a clinical diagnosis. These self-report CCD items could be a viable tool to collect comorbidity data [38].

\section{Conclusions}

There exists an association between CCD and QOL among Chinese GCS, and subjects with CCD generally reported lower QOL scores. These findings warrant the comprehensive cares for GCS. A multidisciplinary team approach and a variety of delivery systems are needed to address the medical, psychosocial, and lifestyle components of gynecological cancer survivorship care.

\section{Availability of data and materials}

Not applicable.

\section{Competing interests}

The authors declare that they have no competing interests.

\section{Authors' contributions}

JW worked on the data analyses, wrote the first draft of the manuscript and made the greatest contribution to the paper. LS, XH, XF, ZT and DH participated in surveying and data analysis. $J$ critically reviewed and 
improved this manuscript. JM was the project coordinator and participated in all parts of the work. ZN and ZP initiated the study. All authors provided critical revision of the paper, and read and approved the final manuscript.

\section{Authors' information}

Not applicable.

\section{Acknowledgements}

We would like to thank the Shanghai Cancer Rehabilitation Club for supporting this study. We are very grateful to all cancer survivors who willingly and generously gave their time.

\section{Funding}

This study was supported by grants from the National Social Science Foundation of China (Award Number 13CSH076) and Shanghai Municipal Commission of Health and Family Planning (Award Number 20134068).

\section{Author details}

${ }^{1}$ School of public health and Key Laboratory of Public Health Safety, Fudan University, 30 Dong-An Road, Shanghai 200032, China. ${ }^{2}$ Jonathan and Karin Fielding School of Public Health, University of California, 650 Charles Young Drive South, Los Angeles, CA 90095, USA. ${ }^{3}$ College of Clinical Medicine, Anhui Medical University, Hefei, Anhui Province, China. ${ }^{4}$ Shanghai Health Education Association, 122 Shan-Xi-Nan Road, 200040 Shanghai, China. ${ }^{5}$ Shanghai Cancer Rehabilitation Club, 2795 Yang-Gao-Zhong Road, Shanghai 200135, China.

\section{Received: 12 December 2014 Accepted: 7 September 2015}

\section{Published online: 25 September 2015}

\section{References}

1. Ferlay J, Bray F, Pasani P, Parkin D. GLOBOCAN 2002: Cancer incidence, mortality and prevalence worldwide. IARC CancerBase No.5 Version 2.0 ed. Lyon: IARC Press; 2004.

2. Reis LAG, Eisner MP, Kosary CL, Hankey BF, Miller BA, Clegg L, et al. SEER cancer statistics review, 1975-2002. Bethesda, MD: National Cancer Institute; 2004.

3. Kim K, Zang R, Choi SC, Ryu SY, Kim JW. Current status of gynecological cancer in China. J Gynecol Oncol. 2009;20(2):72-6.

4. Youlden D, Baade P, Coory M. Cancer survival in Queensland, 2002. Queensland Health and Queensland Cancer Fund: Brisbane, Queensland; 2005.

5. Brenner H, Gondos A, Arndt V. Recent Major Progress in Long-Term Cancer Patient Survival Disclosed by Modeled Period Analysis. J Clin Oncol. 2007;25(22):3274-80.

6. Yue H, Zheng AW. Effects of Postoperative Radiotherapy on the Prognosis of Endometrial Carcinoma. Cancer Res Prevent and Treat. 2001;28(4):308-9.

7. Bodurka DC, Sun CC. Sexual function after gynecologic cancer. Obstet Gynecol Clin North Am. 2006;33(4):621-30

8. Dunton CJ. Management of treatment-related toxicity in advanced ovarian cancer. Oncologist. 2002;7 Suppl 5:11-9.

9. Maas HA, Kruitwagen RF, Lemmens VE, Goey SH, Janssen-Heijnen ML. The influence of age and co-morbidity on treatment and prognosis of ovarian cancer: a population-based study. Gynecol Oncol. 2005;97:104-9.

10. du Bois A, Rochon J, Lamparter C, Pfisterer J. Pattern of care and impact of participation in clinical studies on the outcome in ovarian cancer. Int J Gynecol Cancer. 2005;15:183-91.

11. DiSilvestro P, Peipert JF, Hogan JW, Granai CO. Prognostic value of clinical variables in ovarian cancer. J Clin Epidemiol. 1997;50:501-5.

12. O'Malley CD, Cress RD, Campleman SL, Leiserowitz GS. Survival of Californian women with epithelial ovarian cancer, 1994-1996: a population-based study. Gynecol Oncol. 2003;91:608-15.

13. Janssen-Heijnen ML, Houterman S, Lemmens VE, Louwman MW, Maas HA, Coebergh JW. Prognostic impact of increasing age and comorbidity in cancer patients: a population-based approach. Crit Rev Oncol Hematol. 2005;55:231-40.

14. Vistad I, Fossa SD, Dahl AA. A critical review of patient-rated quality of life studies of long-term survivors of cervical cancer. Gynecol Oncol. 2006;102(3):563-72.
15. Steginga SK, Dunn J. Women's experiences following treatment for gynecologic cancer. Oncol Nurs Forum. 1997;24(8):1403-8.

16. Greimel E, Thiel I, Peintinger F, Cegnar I, Pongratz E. Prospective assessment of quality of life of female cancer patients. Gynecol Oncol. 2002;85(1):140-7.

17. Club SCR: A brief introduction to the Shanghai cancer rehabilitation cancer club. 2014. http://www.shcrc.cn/webs/field_news.aspx?id=35.

18. Aaronson NK, Ahmedzai S, Bergman B, Bullinger M, Cull A, Duez NJ, et al. The European Organization for Research and Treatment of Cancer QLQ-C30: a quality-of-life instrument for use in international clinical trials in oncology. J Natl Cancer Inst. 1993;85(5):365-76.

19. Bottomley A, The EORTC Quality of Life Group. EORTC QLQ-C30 Scoring Manual. 3rd ed. Brussels: EORTC; 2001.

20. Montazeri A, Hole DJ, Milroy R, McEwen J, Gillis CR. Quality of life in lung cancer patients: does socioeconomic status matter? Health Qual Life Outcomes. 2003;1:19.

21. Jordhøy MS, Fayers P, Loge JH, Saltnes T, Ahlner-Elmqvist M, Kaasa S. Quality of life in advanced cancer patients: the impact of sociodemographic and medical characteristics. $\mathrm{Br} J$ Cancer 2001;85:1478-85.

22. Guralnik JM, LaCroix AZ, Everett DF, Kovar M. Aging in the Eighties: The Prevalence of Co-morbidity and Its Association With Disability, vol. 170. Hyattsville, Md: National Center for Health Statistics; Advance Data From Vital and Health Statistics; 1989.

23. Schellevis FG, van der Velden J, van de Lisdonk E, van Eijk JT, van Weel C. Comorbidity of chronic diseases in general practice. J Clin Epidemiol. 1993;46:469-73.

24. Cronin-Fenton DP, Nørgaard M, Jacobsen J, Ewertz M, Lash TL, Sørensen HT. Comorbidity and survival of Danish breast cancer patients from 1995 to 2005. Br J Cancer. 2007;96(9):1462-8.

25. Asmis TR, Ding K, Seymour L, Shepherd FA, Leighl NB, Winton TL, et al. Age and comorbidity as independent prognostic factors in the treatment of non small-cell lung cancer: a review of National Cancer Institute of Canada Clinical Trials Group trials. J Clin Oncol. 2008;26(1):54-9.

26. Sarfati $D$, Hill $S$, Blakely $T$, Robson B, Purdie $G$, Dennett $E$, et al. The effect of comorbidity on the use of adjuvant chemotherapy and survival from colon cancer: a retrospective cohort study. BMC Cancer. 2009;9:116.

27. Akhmedkhanov A, Zeleniuch-Jacquotte A, Toniolo P. Role of exogenous and endogenous hormones in endometrial cancer: review of the evidence and research perspectives. Ann N Y Acad Sci. 2001;943:296.

28. Modesitt SC, van Nagell JR. The impact of obesity on the incidence and treatment of gynecologic cancers: a review. Obstet Gynecol Surv. 2005;60(10):683-92.

29. Kaaks R, Lukanova A, Kurzer MS. Obesity, endogenous hormones, and endometrial cancer risk: a synthetic review. Cancer Epidemiol Biomarkers Prev. 2002;11:1531.

30. Everett E, Tamimi H, Greer B, Swisher E, Paley P, Mandel L, et al. The effect of body mass index on clinical/pathologic features, surgical morbidity, and outcome in patients with endometrial cancer. Gynecol Oncol. 2003;90:150.

31. Eifel PJ, Jhingran A, Bodurka DC, Levenback C, Thames $H$. Correlation of smoking history and other patient characteristics with major complications of pelvic radiation therapy for cervical cancer. J Clin Oncol. 2002;20:3651.

32. Reynolds K, Gu D, Whelton PK, Wu X, Duan X, Mo J, et al. Prevalence and risk factors of overweight and obesity in China. Obesity. 2007;15(1):10-8.

33. Yang ZJ, Liu J, Ge JP, Chen L, Zhao ZG, Yang WY, et al. Prevalence of cardiovascular disease risk factor in the Chinese population: the 2007-2008 China National Diabetes and Metabolic Disorders Study. Eur Heart J. 2012;33:213-20

34. Le Marchand L, Wilkens LR, Kolonel LN, Hankin JH, Lyu LC. Associations of sedentary lifestyle, obesity, smoking, alcohol use, and diabetes with the risk of colorectal cancer. Cancer Res. 1997;57(21):4787-94.

35. Fisher EB, Brownson CA, OToole ML, Shetty G, Anwuri W, Glasgow RE. Ecological approaches to self-management: the case of diabetes. Am J Public Health. 2005;95:1523-35.

36. McCorkle R, Ercolano E, Lazenby M, Schulman-Green D, Schilling LS, Lorig K, et al. Self-Management: Enabling and empowering patients living with cancer as a chronic illness. CA Cancer J Clin. 2011;61:50-62. 
37. Katz JN, Chang LC, Sangha O, Fossel AH, Bates DW. Can comorbidity be measured by questionnaire rather than medical record review? Med Care. 1996;34:73-84.

38. Sridharan S, Berdeprado J, Vilar E, Roberts J, Farrington K. A self-report comorbidity questionnaire for haemodialysis patients. BMC Nephrol. 2014;15:134.

Submit your next manuscript to BioMed Central and take full advantage of:

- Convenient online submission

- Thorough peer review

- No space constraints or color figure charges

- Immediate publication on acceptance

- Inclusion in PubMed, CAS, Scopus and Google Scholar

- Research which is freely available for redistribution 